\title{
LA MATEMÁTICA Y SU INTERRELACIÓN CON EL ARTE Y OTRAS DISCIPLINAS
}

\author{
A MATEMÁTICA E SUA INTER-RELAÇÃO COM A ARTE E OUTRAS \\ DISCIPLINAS
}

\author{
MSc. Elena Fernández de Carrera \\ Directora de la Unidad de Bioestadística Clínica- Facultad de Ciencias Médicas \\ - Universidad Nacional del Litoral- Santa Fe - Argentina. \\ Dra. Ana Palmira Bittencourt Santos Casimiro \\ Programa de Pós-graduação em Memoria: Linguagem e Sociedade (UESB)
}

\section{Resumen}

En este artículo se presenta una visión de la universalidad de la matemática, y su relación con algunas expresiones artísticas. Su presencia se detecta en la cultura de los pueblos, según puede rastrearse desde la antigüedad hasta nuestros días. Lo hace desde un doble aspecto. El primero intenta mostrar cómo descubrir la presencia de la matemática en las manifestaciones de arte; y el segundo, cómo el artista ve la matemática desde su óptica. Se hace hincapié en la arquitectura de la actualidad con arquitectos como Le Corbusier, Oscar Niemeyer, Gaudí y Iannis Xenakis. En verdad, la matemática tiene cierta analogía con todas las formas conocidas de arte, pero también con la ciencia, incluyéndose la medicina.

Palabras Clave: Matemática. Arte. Arquitectura. Interdisciplinaridad.

\section{Resumo}

Neste artigo apresentamos uma visão da universalidade da matemática e sua relação com algumas expressões artísticas. Sua presença é detectada na cultura dos povos, como pode ser traçada desde a Antiguidade até os dias atuais. Isso é feito num duplo aspecto. $\mathrm{O}$ primeiro tenta mostrar como descobrir a presença da matemática nas manifestações artísticas; e o segundo, como o artista enxerga a matemática a partir de sua perspectiva. A ênfase é colocada na arquitetura atual por arquitetos como Le Corbusier, Oscar Niemeyer, Gaudí e Iannis Xenakis. De fato, a matemática tem alguma analogia com todas 
as formas conhecidas de arte, mas também com a ciência, incluindo a medicina.

Palavras-chave: Matemática. Arte. Arquitetura Interdisciplinaridade

\section{Introducción}

El mundo cambia en especial los conocimientos y lo hace con una velocidad cada vez mayor sobre todo como sucede hoy en día que todos ellos están atravesados por la informática. Así los sistemas de numeración nacen en la antigua civilización sumeria, en la Mesopotamia Asiática, allá por los años 4000 a.C. hasta que no hace demasiado tiempo aparecen los números como se los conoce en la actualidad, pero ya a la matemática se la reconocía como una ciencia con profunda interacción e influencia en distintas actividades del hombre, pero siempre interactuando con ellas.

Ya se ha señalado que nadie puede negar la importancia de la matemática para plantear y solucionar problemas. Con una historia extensa y diversa, sus métodos son universales y se valen de herramientas cada vez más evolucionadas. Este artículo presenta, la relación interdisciplinaria entre la matemática y la arquitectura, la necesidad de la primera en áreas aparentemente alejadas del hecho que se está analizando. Así, el enfoque interdisciplinario y el multidisciplinario son fundamentales en el estadio del conocimiento contemporáneo.

\section{Interdisciplinariedad}

La interdisciplinariedad hace su aparición a fines del siglo XX y es precisamente el trabajo mancomunado de varias disciplinas para enfrentar una temática o un problema determinado con la aplicación de varias disciplinas y poder llegar así al entendimiento del tema o a la resolución del problema. Además según Nicolescu (1999, p. 35) también concierne a la transferencia de métodos de una disciplina a la otra, pero hay un hecho muy importante y es que trasciende la investigación disciplinaria.

Edgard Morín señala en su obra la importancia de la interdisciplinariedad

[...] para el entendimiento de la complejidad del conocimiento humano, se debe agregar, que la rapidez de los cambios y los estudios cada vez más

\begin{tabular}{l|l} 
Revista RBBA & Revista Binacional Brasil Argentina
\end{tabular} 
pormenorizados hacen que los distintos enfoques científicos deban aunarse para la comprensión de un problema (apud CARRERA, 2013).

La transdisciplinariedad concierne como el prefijo "trans" lo indica, lo que está a la vez entre las disciplinas, a través de las diferentes disciplinas y más allá de toda disciplina. Su finalidad es la comprensión del mundo presente en el cual uno de los imperativos es la unidad del conocimiento (NICOLESCU, 1999, p.35).

Según el mismo Nicolescu (1999),

la disciplinariedad, la pluridisciplinariedad, la interdisciplinariedad y la transdisciplinariedad son las cuatro flechas de un solo y mismo arco: el del conocimiento. Además afirma: $\mathrm{Si}$ la multidisciplinariedad y la interdisciplinariedad refuerzan el diálogo entre dos culturas, la transdisciplinariedad permite vislumbrar su unificación abierta. Las consideraciones precedentes sobre los niveles de realidad, de percepción y de representación, más allá del ejemplo del arte y de la ciencia, ofrecen una base metodológica de la conciliación de dos culturas artificialmente antagonistas - la cultura científica y la cultura humanista - por su superación en la unidad abierta de la cultura transdisciplinaria (p.37).

Por eso en este artículo se continuará con la relación de la matemática con la arquitectura y se mencionarán algunas más con otras ciencias. ¿Por qué si la matemática está entrelazada con todas las ciencias, con todas las artes y en todos los tiempo, su aprendizaje fue y es tan dificultoso sobre todo hoy? (CARRERA, 2013 p. 116) Porque hay que cambiar los modos de enseñar para que el aprendizaje sea eficaz y placentero.

Es obvio que el porvenir no se conoce, pero al ritmo que avanza el conocimiento y la tecnología en especial la informática, es evidente que será cambiante y complicado. Por ello todo aquél que esté relacionado con la educación debe preparar a las generaciones para este mundo que se vislumbra. El educador debe ser especial. Debe transformar a los estudiantes en pensadores, sobre todo cuestionadores de aquello que se acepta como verdad, coincidiendo así con Tomás Abraham (2012) cuando afirma que la verdad es el valor que legitima una autoridad (p. 10)

La enseñanza se debe basar en la resolución de problemas que no son un conjunto de enunciados sin sentido donde sólo se deben realizar operaciones algorítmicamente, "la problematización es algo más complejo”. Según Foucault (2005, p. 97) “analizar la problematización es analizar su proceso" y es precisamente analizando problemas que se puede estatuir que elementos resultan importantes para la misma. 
Cuando se forma al alumno en la resolución de problemas o sea en la heurística que es la base del proceso creativo en matemática y que en la primera mitad del siglo XX fue rescatado del olvido por George Polya (1887-1985) quien afirmó que Euclides tuvo razón cuando señaló que la matemática es una ciencia sistemática y conductiva, pero también experimental e inductiva.

Se dice todo esto porque es obvio que se está inmerso en el medio de una crisis educativa que no deja de ser importante y de estar vigente, pero que es cambiante como la realidad social. Se está en un mundo cambiante, donde ya Michael Foucault aseguraba que nunca pasa lo mismo, el orden del acontecimiento es el de la multiplicidad contingente siempre renovada e inconclusa (apud ABRAHAM, 2002, p. 96). No existen verdades permanentes ni historia continua. Por esta razón se estudia el presente y el proceso de construcción del sujeto actual porque sólo comprendiendo como se llegó a ser lo que se es en la actualidad se puede comprender lo que se es y además llegar a ser.

\section{Arte, matemática, arquitectura}

Por otra parte, también el arte es una forma de lenguaje universal y cada pueblo, cultura y hábitat determinan y/o propician el modo como suceden las expresiones artísticas, lo que dependerá de algunas singularidades como clima, lengua, costumbres, etc. Un buen ejemplo es la arquitectura, que queda casi subordinada al material constructivo del que dispone una determinada región

La Matemática y el arte se encuentran muy relacionados y en especial con la arquitectura y no se puede dudar de su vinculación estrecha con las humanidades y con las otras ciencias. Desde los primeros vestigios de las civilizaciones, desde las más antiguas se encuentran pruebas que relacionan a la matemática con la arquitectura.

Esta relación se remonta a los orígenes del hombre en el planeta y se justifica por la propia relación que la matemática tiene, por un lado, con la naturaleza y sus fenómenos, y por otro lado, con el origen del arte que también ocurrió debido a la observación de la naturaleza, lo que fue desarrollando, paulatinamente, en el hombre, el sentido estético y el conocimiento de las categorías artísticas. Esto quiere decir que, en la medida en que el hombre se familiarizaba con el pulsar de la naturaleza, poco a poco asimilaba y memorizaba visiones estéticas como el brillo del pelaje de los animales, las formas geométricas de las piedras, la

\begin{tabular}{l|l} 
Revista RBBA & Revista Binacional Brasil Argentina
\end{tabular} 
simetría de algunas plantas, el ritmo de las olas y del nacimiento y puesta del sol y la variedad de colores de las flores y los frutos (FISCHER, 1966).

Así, mucho antes que la ciencia dividiera el conocimiento en disciplinas, algunas categorías se volvieron comunes a varias áreas del saber, empezando por las categorías de "tiempo y espacio". Advinieron de ellas provienen otras categorías derivadas de las de tiempo y espacio, a saber: forma, tamaño, distancia, peso, ubicación espacial, color, simetría, proporción, armonía, ritmo, aislamiento, entre otras. Tales categorías se encuentran en muchos sectores del conocimiento y explican la razón fundamental entre las matemáticas y el arte, así como, entre las matemáticas y otras ciencias y, en el caso de las artes, entre sí.

Al recorrer la historia de la Antigüedad Clásica se encuentra a la matemática entrelazada con todas las manifestaciones del hombre: la filosofía, el arte, la arquitectura. Sólo basta ver los monumentos griegos de la época, tal como el Partenón, distinguir las magníficas proporciones que respetaban en su arquitectura, así como en sus estatuas. La proporción áurea era la predominante. Donde la razón de proporcionalidad era el célebre número de oro al que se simboliza con la letra griega $\varphi$. Y estas proporciones son las proporciones áureas. Esta fascinación por la proporción continúa hasta que en el Medievo se calculó su valor aproximado que es $\varphi=1,61803 \ldots \ldots$

Este número ya mítico, que en la actualidad forma parte de libros, películas y conversaciones en general rigió las proporciones en la arquitectura, la escultura y las artes en general desde la Grecia antigua. Leonardo da Vinci (1452 - 1519), reconocido pintor que era también ingeniero, anatomista (y uno de los más grandes anatomistas de la historia), inventor, científico y poeta, incursionaba en cada una de las actividades mencionadas demostrando su dominio de la matemática, de las proporciones y también de los códigos. Leonardo aplica esta sección aurea y es notorio en sus pinturas, en La Virgen de las Rocas, en el inolvidable cuadro de la Gioconda y en la imagen del hombre de Vitruvio que permite visualizar las proporciones del cuerpo humano que fueron fijadas por Luca Pacioli que estudió y concretó las proporciones que debía tener un cuerpo humano.

Ya más adelante en el tiempo y en la arquitectura, muy asociada a la geometría aparece la imagen del genial arquitecto catalán Antoni Gaudí (1852- 1926), que según Claudí Alsina (2008) se proclamaba geómetra no obstante también proclamaba que las expresiones algébricas lo único que hacen es complicar (p. 145) pero el amor de Gaudí por la geometría 
es innegable como puede apreciarse en todas sus obras, en especial en la Iglesia de la Sagrada Familia en Barcelona, España. En la Figura 1 puede verse su frente donde el trabajo es importantísimo y donde además se hace necesario mencionar las puertas de ingreso que no son de madera común sino con un trabajo en metal pintado de verde simulando ¡ramas verdes de hiedra con unos escarabajos en marrón! (figura 2).

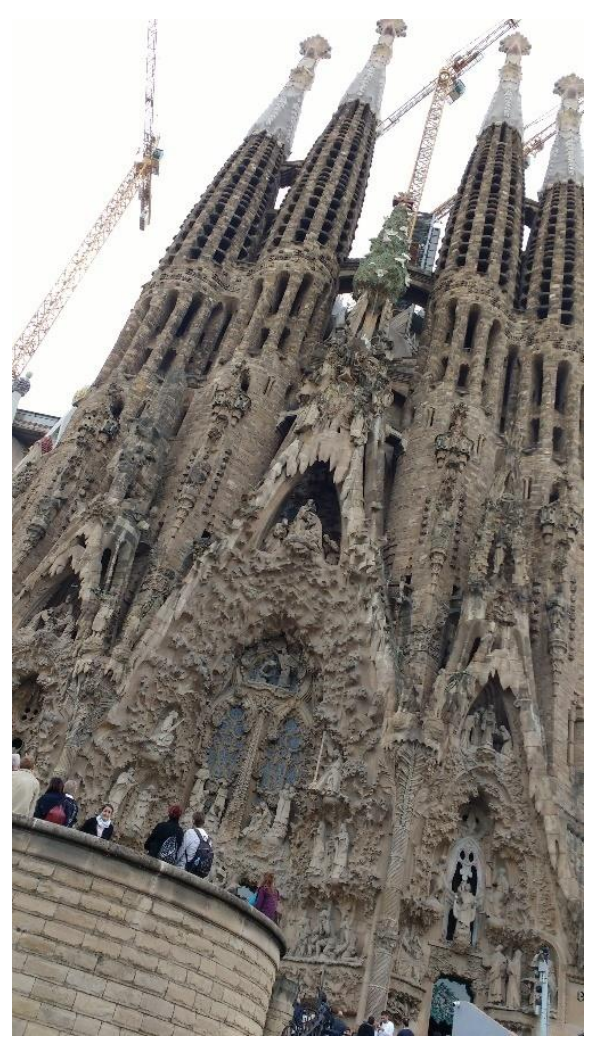

Figura 1. Frente Sagrada Familia. Barcelona

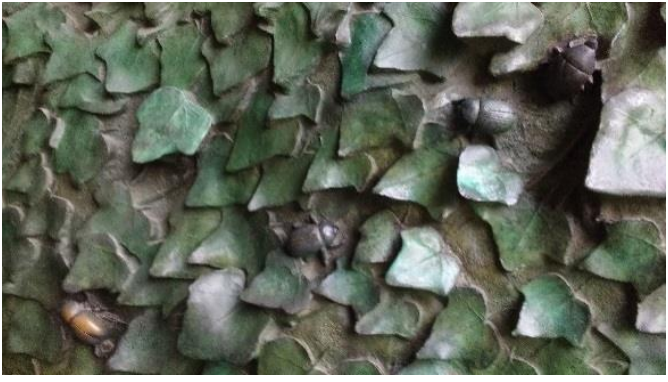

Figura 2. Detalles puerta ingreso Sagrada Familia

Pero mencionando la arquitectura y el número de oro $\varphi$ así como el hombre de Vitruvio no puede dejar de citarse a Charles-Édouard Jeanneret-Gris (1887 - 1965) más conocido por Le Corbusier, seudónimo que adoptó pero que en realidad era el apellido de su abuelo, arquitecto de origen suizo nacionalizado francés. Pero además fue un pintor notable y con gran influencia teórica en la arquitectura que proyectó y construyó casas y hasta grandes urbanizaciones. Era un admirador de la obra de Gaudí.

Le Corbusier tenía gran preocupación por las proporciones y en especial por las del hombre por lo cual se puso a estudiarlas y a normalizarlas para su uso en muebles y distintos 
elementos que se fabrican para el uso de las personas. Creo así El Modulor normalización realizada en base a las medidas de un hombre cuya altura aproximada era de 1,83 $\mathrm{m}$.

Muchas obras de Le Corbusier fueron declaradas Patrimonio de la Humanidad, en distintos países del mundo. En Argentina en la ciudad de La Plata existe una casa diseñada por él, la casa Curuchet magnifica, de líneas rectas, cuyas ventanas juegan con la luz del día y son una visión panorámica del exterior.

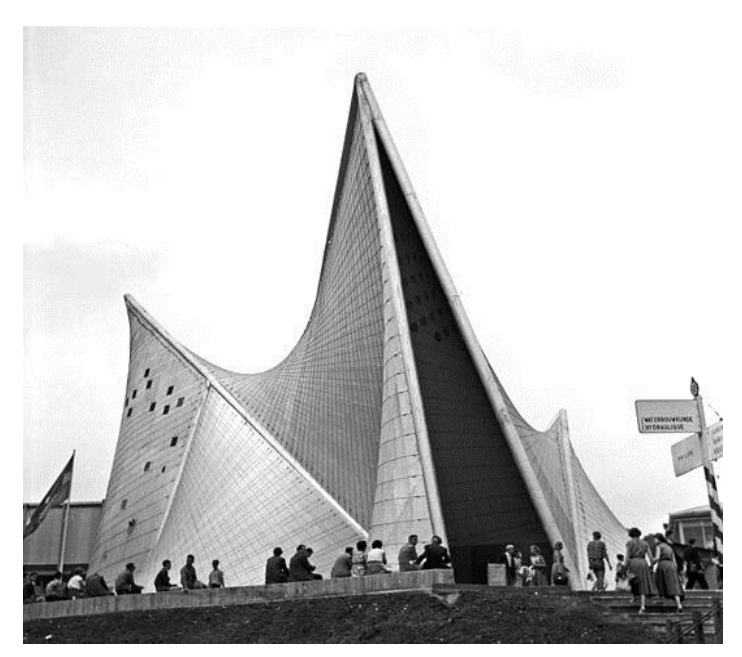

Figura 3. Pabellón Phillips.

En la Exposición Universal de Bruselas del año 1958, Le Corbusier invitó al ingeniero de origen griego Iannis Xenakis (1922 - 2001) para trabajar juntos en el diseño y construcción del pabellón Phillips, donde predominan las curvas hiperbólicas que maravillan la visión de los visitantes que fueron obtenidas a partir de la resolución de una simple ecuación matemática si bien Xenakis trascendió más como músico. Su música es la llamada "música estocástica" que está fundamentada en la teoría de las probabilidades y la teoría matemática de juegos y que a partir del año en que apareció 1954 fue la base de la música electrónica.

Le Corbusier trabajó con el célebre arquitecto brasileño Oscar Niemeyer (1907 2012), que diseñó y levantó la ciudad de Brasilia, y que junto a Le Corbusier diseñaron y construyeron el edificio de las Naciones Unidas en Nueva York en cuya fachada se identifican tres rectángulos áureos. La Catedral de Brasilia es un claro ejemplo de las curvas bellas que proponen en arquitectura ambos artistas. 


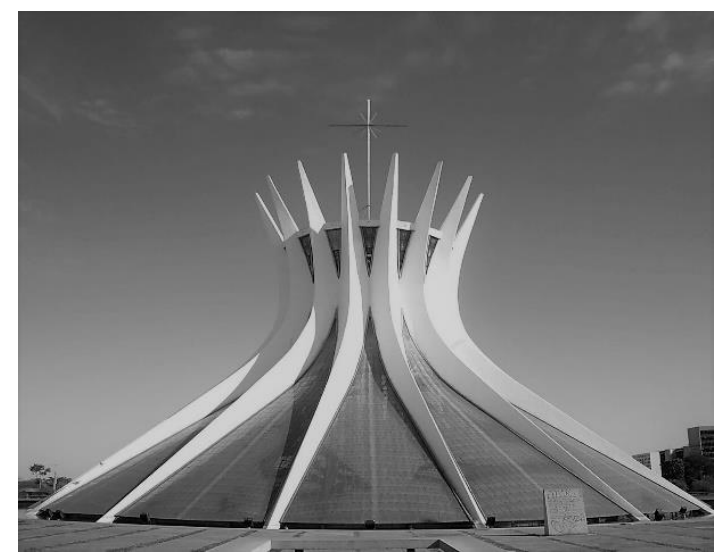

Figura 4. Catedral de Brasilia

Quedan aún varios temas matemáticos por ver que tienen una importancia sin igual en el mundo actual pero que trataremos en un próximo artículo. Entre ellos la teoría del caos, los fractales que no sólo tienen importancia en el arte, como en las pinturas de Pollock, sino también en la medicina donde los fractales resultan hoy fundamentales en la interpretación de las imágenes de distintos tipos de cáncer.

Citamos algunos artistas y obras de arte, como ejemplos variados en el tiempo y el espacio. Como se ha dicho inicialmente, observamos que, en los ejemplos, es posible determinar las mencionadas categorías "tiempo" y "espacio" y, especialmente, las categorías derivadas de esas iniciales las cuales están presentes tanto en el arte como en la matemática. De ahí, establecemos ciertas relaciones pertinentes y conceptos utilizados por las dos áreas del saber.

En las artes plásticas utilizamos comúnmente las categorías forma, tamaño, distancia, peso, ubicación espacial, diagonalidad, verticalidad, horizontalidad, transparencia, profundidad, claro-oscuro, color, textura, equilibrio, simetría, proporción, armonía, ritmo, aislamiento; En la arquitectura usamos casi todas esas y otras más, a saber, la función, el programa y el partido, así como la estructura de los materiales y el cálculo; En la poesía y en la literatura utilizamos muchas de las ya mencionadas y otras más: la proporción, la métrica, la aliteración, entre otras (WÖLFFLIN, 1984).

El teatro y la danza traducen partes, colores, ritmo, armonía, tiempo, ubicación, escenografía, así como la música; Y la música, propiamente dicha, se expresa mediante el sonido, el eco, el silencio, el ritmo, la armonía, la variación, además de otras categorías. La fotografía, el arte cinematográfico y la TV son productos de una nueva era tecnológica con 
paradigma diferente de las otras expresiones artísticas aquí tratadas. Pero en estas tres últimas, quedan evidentes los conocimientos y el uso de las categorías, plano, espacio, tridimensionalidad, foco, perspectiva, color, lateralidad, tiempo, sólo se repiten las más constantes.

Además, observamos que la relación entre el arte y las matemáticas no se limita a las condiciones del mundo material. En el plano de las ideas, la matemática utiliza herramientas extraídas del campo de la filosofía y de otras ciencias como la lógica, la estética, el método científico, la física y la astronomía, como ejemplos, y que el arte, a su vez, como recurso y propulsión usa algunas categorías psicológicas y espirituales fundamentales para la creación humana, como éxtasis, arrebatamiento, interés intrínseco, miedo, repulsión, encantamiento, sentimiento de plenitud, placer estético, paz, belicosidad (WÖLFFLIN, 1984).

Al concluir este texto, subrayamos que las referidas categorías se encuentran en muchos sectores del conocimiento, éstas explican la razón fundamental de la semejanza que existe entra la matemática y el arte, así como, entre la matemática y otras ciencias y, entre sí, en el caso de las artes. Señalamos que, en el estudio de las relaciones propuestas observamos las semejanzas y analogías, pero también las diferencias. El arte no es utilitario. Éste provoca, instiga, cuestiona, pero no intenta responder a interrogantes, por lo menos racionalmente. La Matemática intenta solucionar, de modo racional, las cuestiones enigmáticas o dudas propuestas. Ésta es quizás la fuerza motriz más notable del conocimiento. Finalmente concluimos que, si la función principal de la matemática es solucionar, objetivando el avance de la ciencia, a su vez, el arte, es la creación libre del espíritu y su motivación transita por instancias de la mente cuyos estudios escapan a nuestro propósito.

\section{Referencias}

ABRAHAM, Tomás. La lechuza y el caracol. Contrarrelato político. Buenos Aires: Sudamericana., 2012.

ALSINA, Claudí. 2008. El club de la hipotenusa. Ed. Ariel S.A. Barcelona. España.

CARRERA, Elena F. Matemática, matemática... ¡Cuántos problemas traes! ¡y cuántas soluciones nos ofreces! Libro: Acercarse a la investigación Científica, reflexiones y propuestas didácticas. Laborde libros 2013. 
CORBALÁN, Fernando. La proporción áurea. El lenguaje matemático de la belleza. España: EDITEC, 2010.

FISCHER, Ernst. A Necessidade da Arte. Rio de Janeiro: Zahar, 1966. 255 p.

FOUCAULT, M. La verdad y las formas jurídicas. 2. ed. Barcelona: Gedisa S.A; Corregida, 2003.

FOUCAULT, Michel. Las palabras y las cosas. Una arqueología de las ciencias Humanas, 2005. (Siglo XXI Editores Argentina S. A).

FRANCASTEL, Pierre. A Realidade Figurativa: elementos estruturais de sociologia da arte. São Paulo: Perspectiva; EDUSP, 1973. 447p. (Estudios, 21).

Martins Fontes, 1984, 278p. (Siglo XXI Editores Argentina S. A).

MORÍN, Edgar. Los siete saberes necesarios para la educación del futuro. Buenos Aires: Nueva Visión, 2009.

NICOLESCU, B. La transdisciplinariedad. Manifiesto. Ed. Du Rocher, 1999.

STEWART, Ian. Historia de las matemáticas en lo últimos 10.000 años. Barcelona. España: Drakontos; Critica, 2008.

WÖLFFLIN, Heinrich. Conceitos Fundamentais de História da Arte. São Paulo, 1984, 278p.

\section{Sobre as autoras}

Elena Fernández de Carrera. Profesora de Matemática del Instituto del Profesorado de la Universidad Nacional del Litoral (UNL), de la Licenciatura en Matemática Aplicada y de la Facultad de Ing. Química de la UNL. Master Science Biometría por la Escuela de Graduados Fac. de Agronomía Universidad de Buenos Aires. Profesora Titular con dedicación exclusiva. Directora del Departamento de Matemática de la Facultad de Bioquímica y Cs. Biológicas de la UNL. Autora de diversas publicaciones en el ámbito nacional e internacional. Correo Electrónico: elenacarrera2@gmail.com

Ana Palmira Bittencourt Santos Casimiro. Professora Plena da Universidade Estadual do Sudoeste da Bahia (UESB) e Professora do Programa de Pós-Graduação em Memória, Linguagem e Sociedade (PPGMLS-UESB). Doutora em Educação pela UFBA e Pós-Doutora em Educação pela UNICAMP. É pesquisadora vinculada ao Grupo de Estudos e Pesquisas História, Educação e 
Sociedade no Brasil - HISTEDBR e ao Museu Pedagógico, no qual coordena o Grupo Fundamentos da Educação: Memória, Imagem, Religião. É autora de publicações na área de História da Arte e História da Educação, com ênfase em barroco e Brasil Colonial. Endereço Eletrônico: anapalmira32@gmail.com 